\title{
REFINEMENTS OF THE HADAMARD AND CAUCHY-SCHWARZ INEQUALITIES WITH TWO INEQUALITIES OF THE PRINCIPAL ANGLES
}

\author{
HUAMIN ZHANG AND HONGCAI YIN
}

\begin{abstract}
By discussing two volume formulae for the parallelotope, some refinements of the Hadamard and Cauchy-Schwarz inequalities are given and a class of principal inequalities related a parallelotope is established. This class of principal inequalities have a close relation to the Hadamard and Fischer determinant inequalities. By using the interlacing property, a principal inequality related to two subspaces is given which has a close relation to the Koteljanskii determinant inequality. Analysis indicates that these two principal inequalities can be extended to two class of principal inequalities easily.
\end{abstract}

Mathematics subject classification (2010): 51M16, 51M20.

Keywords and phrases: Parallelotope volume formula, Hadamard inequality, Cauchy-Schwarz inequality, principal angle inequality.

\section{REFERENCES}

[1] H. M. ZhANG, S. G. YANG, A new volume formula for a simplex, Journal of Zhejiang University (Science Edition) 35 (1) (2008) 5-7.

[2] G. S. Leng, Y. Zhang, B. L. MA, Largest parallelotopes contained in simplices, Discrete Mathematics, 211 (2000) 111-123.

[3] E. Gover, N. KRIKORIAN, Determinants and the volumes of parallelotopes and zonotopes, Linear Algebra and its Applications 433 (1) (2010) 28-40.

[4] A. Ben-IsRael, A volume associated with $m \times n$ matrices, Linear Algebra and Its Applications, 164 (1992) 87-111.

[5] H. X. LI, G. S. LENG, A matrix inequality with weights and its applications, Linear Algebra and Its Applications, 185 (1993) 273-278.

[6] G. S. LENG, Y. ZHANG, The generalized sine theorem and inequalities for simplices, Linear Algebra and Its Applications, 278 (1-3) (1998) 237-247.

[7] H. C. Yin, H. M. Zhang, Properties of bisection planes of dihedral angles of a simplex in the $n$ dimensional Euclidean space, Journal of Zhejiang University (Science Edition) 39 (1) (2012) 18-19.

[8] J. Sheng, Angles between Euclidean subspaces, Geometriae Dedicata 63 (2) (1996) 113-121.

[9] B. Zhou, Z. Y. LI, G. R. DUAN, Y. WANG, Weighted least squares solutions to general coupled Sylvester matrix equations, Journal of Computational and Applied Mathematics 224 (2) (2009) 759776.

[10] A. G. WU, L. L. LV, G. R. DUAN, Iterative algorithms for solving a class of complex conjugate and transpose matrix equations, Applied Mathematics and Computation 217 (21) (2011) 8343-8353.

[11] H. M. ZHANG, Reduced-rank gradient-based algorithms for generalized coupled Sylvester matrix equations and its applications, Computers \& Mathematics with Applications 70 (8) (2015) 20492062.

[12] Zhagn H M, Ding F., Iterative algorithms for $X+A^{\mathrm{T}} X^{-1} A=I$ by using the hierarchical identification principle, Journal of the Franklin Institute, 2016, 353 (5): 1132-1146.

[13] Z. Y. LI, B. Zhou, Z. L. LI, On exponential stability of integral delay systems, Automatica 49(11) (2013) 3368-3376. 
[14] B. Zhou, W. X. Zheng, G. R. DuAn, Stability and stabilization of discrete-time periodic linear systems with actuator saturation, Automatica 47(8) (2011) 1813-1820.

[15] R. A. Horn, N. H. RHEe, S. WAsin, Eigenvalue inequalities and equalities, Linear Algebra and its Applications 270 (1-3) (1998) 29-44.

[16] Q. K. Kong, A. ZettL, The study of Jacobi and cyclic Jacobi matrix eigenvalue problems using Sturm-Liouville theory, Linear Algebra and its Applications 434(7) (2011) 1648-1655.

[17] H. SCHULZ-BALDES, Sturm intersection theory for periodic Jacobi matrices and linear Hamiltonian systems, Linear Algebra and its Applications 436(3) (2012) 498-515.

[18] D. M. Zhou, G. L. Chen, G. X. Wu, X. Y. Zhang, On some new bounds for eigenvalues of the Hadamard product and the Fan product of matrices, Linear Algebra and its Applications 438(3) (2013) 1415-1426.

[19] X. Z. ZHAN, On some matrix inequalities, Linear Algebra and its Applications 376(1) (2004) 299 303.

[20] J. M. Miao, A. Ben-IsRael, On principal angles between subspaces in $\mathbb{R}^{n}$, Linear Algebra and Its Applications, 171 (1992) 81-98.

[21] G. H. Golub, C. F. Van Loan, Matrix Computations, 3rd ed., Baltimore, MD: Johns Hopkins University Press, 1996.

[22] X. D. Zhang, Matrix Analysis and Applications, Beijing: Tsinghua University Press, 2004.

[23] R. Bellman, Introduction to Matrix Analysis, Newyork: Mcgraw-Hill Book Company, 1970.

[24] C. Lupu, D. SCHWARZ, Another lool at some new Cauchy-Schwarz type inner product inequalities, Applied Mathematics and Computation 231 (2014) 463-477.

[25] Z. Z. YAN, A unified version of Cauchy-Schwarz and Wielandt inequalities, Linear Algebra and its Applications 428(8-9) (2008) 2079-2084.

[26] R. A. Horn, R. MATHIAs, Cauchy-Schwarz inequalities associated with positive semidefinite matrices, Linear Algebra and its Applications 142 (1990) 63-82.

[27] D. R. Jocić, S. MiLošEvić, Refinements of operator Cauchy-Schwarz and Minkowski inequalities for p-modified norms and related norm inequalities, Linear Algebra and its Applications 488 (2016) 284-301.

[28] S. H. WADA, On some refinement of the Cauchy-Schwarz inequality, Linear Algebra and its Applications 420 (2007) 433-440.

[29] J. M. Aldaz, S. Barza, M. Fujil, M. S. Moslehian, Advances in operator Cauchy-Schwarz inequalities and their reverses, Annals of Functional Analysis, 6 (3) (2015) 275-295.

[30] H. M. ZHANG, F. Ding, A property of the eigenvalues of the symmetric positive definite matrix and the iterative algorithm for coupled Sylvester matrix equations, Journal of the Franklin Institute 351 (1) (2014) 340-357. 